\title{
CRISPR/Cas9 targeting of MCPyV T antigen in Merkel tumors
}

Authors: Reety Arora*\#1, Komal Gupta*1,2,3 ${ }^{*}$ Anjali Vijaykumar ${ }^{1,4}$

Affiliations:

1. National Centre for Biological Sciences, TIFR, Bangalore, India

2. Indian Institute of Science Education and Research, Pune, India

3. MRC Cancer Unit, University of Cambridge, UK

4. Imperial College London, London, UK

\section{${ }^{*}$-Equally contributing shared first authors \\ \#- corresponding author - Reety Arora}

\section{Email ID and address:}

1. Komal Gupta (kg463@cam.ac.uk)

2. Dr. Reety Arora (Corresponding author) (reetya@ncbs.res.in)

\section{ABSTRACT}

Merkel cell carcinoma (MCC) is a rare, aggressive skin cancer caused either by Merkel cell polyomavirus (MCPyV) T antigen gene expression, post integration ( $80 \%$ cases), or by UV mediated DNA damage. Viralpositive Merkel tumors are not only caused by but also oncogenically addicted to tumor antigen expression. In this study we used CRISPR-Cas9 based gene-editing to develop a potential therapeutic tool for MCPyV positive MCC. CRISPR (Clustered Regularly Interspaced Short Palindromic Repeats)/Cas system is a genome editing technology whereby a guide RNA (gRNA) molecule, targets a Cas endonuclease to a specific genomic site, using sequence homology, and induces a double strand break. To target MCPyV T antigens, we designed a strategy using 2 gRNAs targeting the $T$ antigen genomic region that would cut off a substantial portion of the gene thereby rendering it dysfunctional. We validated the MCPYV T antigen targeting efficiency of our gRNAs, both individually and together by in vitro cleavage assays. Finally, to translate this finding, we delivered this CRISPR system in patient-derived MCC cell lines and show reduction in T antigen gene expression. Our proofof-concept study shows that 2 MCPyV targeting CRISPR/Cas gRNAs in combination can knock out MCPyV T antigen, thus, being of therapeutic importance. We hope that this CRISPR system can be potentially delivered in vivo for advancing MCPyV positive MCC treatment in the future. 


\section{INTRODUCTION}

Merkel Cell Carcinoma (MCC) is a rare and aggressive neuroendocrine cancer of the skin ${ }^{1}$. Prolonged UV exposure, advanced age and loss of immune competence are risk factors for this cancer ${ }^{2,3}$. It is either caused by Merkel cell polyomavirus (MCPyV) ${ }^{4}$ or long term Ultraviolet (UV) exposure ${ }^{5-7}$. In the majority of human population MCPyV is a harmless, asymptomatic and life-long infection. However, MCPyV initiates an aggressive cancer if it gets integrated into the susceptible host genome and acquires viral mutations that lead to replication incompetence ${ }^{4}$. In tumors, MCPyV expresses 2 oncoproteins: the small and large Tumor antigens $^{3,4}$. These $\mathrm{T}$ antigens drive tumorigenesis by targeting various tumor suppressors and regulating transcription $^{8-10}$.

A majority of MCC cases have been found to be MCPyV positive ( $60-80 \%)^{1,3,4}$. In virally caused cancers the T antigens are the major drivers in tumor development. Interestingly, T antigen knockdown in MCC cell lines initiates cell cycle arrest and cell death ${ }^{9,11}$. As MCPyV positive MCC tumors are oncogenically addicted to the expression of $\mathrm{T}$ antigens, they are attractive targets for therapeutics.

In this study we developed a CRISPR Cas9 ${ }^{12}$ system to knock-down MCPyV T antigen expression. This is the first report to use CRISPR Cas9 to reduce MCPYV T antigen expression and we hope that with further validation and analysis this will serve as a potential targeted gene therapy system in the future.

The CRISPR (Clustered Regularly Interspaced Short Palindromic Repeats)/Cas9 system is a programmable gene editing system adapted from the bacterial immune system ${ }^{12}$. CRISPR uses a guide RNA (gRNA) molecule that targets a Cas endonuclease to a specific genomic site using sequence homology and PAM (Protospacer adjacent motif) recognition ${ }^{13-15}$. Thus, the end result of Cas9-mediated DNA targeting is a double stranded break (DSB) within the target. This break is then corrected by the cell, usually via the mutagenic Non homologous end joining (NHEJ) pathway, resulting in disruption of target gene ${ }^{16}$. Using transfection or lentiviral transduction, it is possible to induce the cleavage of specific sequences in the human genome by the expression of SpCas9 and a sgRNA molecule. Gene inactivation using this system is highly efficient, and SpCas9 has been previously used to target various genes including HIV proteins and E6/E7 oncoproteins in cervical carcinoma $^{17-19}$.

Here we report the use of the CRISPR SpCas9 system to target the T antigen oncogenes in MCPyV positive MCC cell lines. First, we designed several gRNAs and tested their ability to target $\mathrm{T}$ antigen expression. Then, we designed a strategy to use 2 validated gRNAs and measured their efficiency using an in vitro cleavage assays. Further, we used these two gRNAs together to target the T antigen loci in cells expressing MCPyV T antigens and Cas9. Our study is the first to report the use of the CRISPR-Cas9 system for targeting MCPyV T antigen genes in Merkel tumors. 


\section{MATERIALS AND METHODS}

\section{sgRNA design.}

13 gRNAs were designed to target the small and large Tumor antigen genes of MCPyV. Three CRISPR design tools: Broad institute software (broadinstitute.org/gpp/public/analysis-tools/sgrna-design), CRISPR-MIT (http://crispr.mit.edu) and CRISPOR were used. MKL-1 (Genbank Accession \#: FJ173815.1) and MS-1 (Genbank Accession \#: JX045709.1) MCPyV sequences were used as target DNA sequences. gRNAs with highest specificity score and with lowest possible off targets were selected. Synthetic gRNA oligonucleotides were cloned into pLentiCRISPRv2 (Addgene \#52961) at BsmBI (\# R0580S, NEB) restriction sites. Plasmid DNA was prepared using Stbl3 competent cells (\#C7373-03, Invitrogen) and Qiagen midi prep kits (\#12143).

\section{Synthesis of sgRNAs using IVT.}

Two oligonucleotides were designed as DNA templates, per gRNA, for in vitro transcription (IVT). Forward oligo for Cas9 gRNA synthesis consisted of the T7 promoter (TAATACGACTCACTATAGG), guide RNA, and start of Cas9 gRNA scaffold. Reverse oligo consisted of complete gRNA scaffold with complementarity to 15nt in the forward oligo (shown below). Three G's were added at the gRNA start for efficient T7 transcription.

Target substrate for C3 gRNA in vitro cleavage (TARGET / pam):

5' GATGAAAGCTGCTTTCAAAAGAAGCTGCTTAAAGCATCACCCTGATAAAGgggGAAATCCTGTTAT 3' crRNA oligo for C3 gRNA in vitro transcription (T7 / ADDED G / GUIDE / SCAFFOLD):

Forward primer: TAATACGACTCACTATAGGGAAAGCATCACCCTGATAAAGGTTTTAGAGCTAGAA

Reverse primer: AAAAGCACCGACTCGGTGCCACTTTTTCAAGTTGATAACGGACTAGCCTTATTTTAA

\section{CTTGCTATTTCTAGCTCTAAAAC}

Target substrate for C13 in vitro cleavage (TARGET / pam):

5' CCAGTGTACCTAGAAATTCTTCCAGAACGGATGGCACCTgggAGGATCTCTTCTGCGATGAATC 3'

crRNA oligo for C13 gRNA in vitro transcription (T7 / ADDED G / GUIDE / SCAFFOLD):

Forward primer: TAATACGACTCACTATAGGGTTCCAGAACGGATGGCACCTGTTTTAGAGCTAGAA

Reverse primer: AAAAGCACCGACTCGGTGCCACTTTTTCAAGTTGATAACGGACTAGCCTTATTTTAA

CTTGCTATTTCTAGCTCTAAAAC

Templates for IVT were amplified by the following PCR reaction: Denaturation at $95^{\circ} \mathrm{C}$ for 3 mins followed by 30 cycles of $95^{\circ} \mathrm{C}-30 \mathrm{~s}, 45^{\circ} \mathrm{C}-30 \mathrm{~s}$ and $72^{\circ} \mathrm{C}$ for $30 \mathrm{~s}$ and final annealing at $72^{\circ} \mathrm{C}$ for $7 \mathrm{~min}$. The PCR products were cleaned up using PCR clean up kit (\# A9282, Promega). sgRNAs were synthesized using the MEGAShortScript ${ }^{\mathrm{TM}}$ Kit (\# AM1354, Invitrogen) following the manufacturer's protocol with 2 ug PCR product template over night at $37^{\circ} \mathrm{C}$. The RNA was treated with $1 \mathrm{ul}$ of DNase TURBO for 15 minutes followed by purification using the MEGAclear Transcription Clean-Up Kit (\# AM1908, Invitrogen).

\section{In vitro cleavage assay.}

In vitro cleavage reaction was performed with purified SpCas9 protein (500ng) (Kind gift from Dr. Praveen Vemula's Laboratory) at $37^{\circ} \mathrm{C}$ in cleavage buffer consisting of $20 \mathrm{mM} \mathrm{HEPES}(\mathrm{pH} 7.5), 150 \mathrm{mM} \mathrm{KCl}, 10 \mathrm{mM}$ 
$\mathrm{MgCl} 2,1 \%$ glycerol and $0.5 \mathrm{mM}$ DTT for 1 hour. $300 \mathrm{ng}$ of sgRNA and $200 \mathrm{ng}$ of the target DNA were used for the reaction. PCR amplicons of ST and LT gene regions from plasmid RAZ1 (Addgene \#114381) were used as target DNA templates (Refer Supplementary Table 1 for primers). The PCR amplicon was purified using Ampure beads (\#A63881, Beckman Coulter) as per manufacturer's protocol. The IVC reactions were stopped by treatment with $2 \mathrm{ul}$ of Proteinase $\mathrm{K}(10 \mathrm{mg} / \mathrm{ml})$ at $55^{\circ} \mathrm{C}$ for 30 minute. $1 \mathrm{x}$ gel loading dye was added to the reactions and samples were run on $2 \%$ agarose gels (\# RM273, HIMEDIA).

\section{Plasmid Construction.}

We cloned the NCCR and T antigen derived from MKL-1 into a pLentiCMV backbone vector We used the target region PCR amplicon as the DNA substrate.

\section{Gibson Cloning the gRNAs in pDECKO mCherry.}

For expressing the gRNAs in MCPyV positive cell lines, C3 and C13 were cloned into pDECKO mCherry lentiviral plasmid using Gibson assembly. The backbone plasmid (kind gift of Dr. Debjyoti Chakroborty, IGIB) was digested using $\mathrm{BsmBI}$ at $55^{\circ} \mathrm{C}$ for 2 hours. The cut plasmid was run on a $0.8 \%$ agarose gel and the linear band was gel extracted. The oligonucleotides for insert 1 consisting of the gRNAs, T7 and part of $\mathrm{H} 1$ promoter and gRNA scaffold were designed using http://crispeta.crg.eu. Insert 1 was cloned into digested vector and was transformed into Stbl 3 competent cells. Positive clones were selected using colony PCR followed by plasmid isolation. This intermediate plasmid was again digested by BsmB1. The constant insert 2 consisting of remaining part of gRNA 1 scaffold and $\mathrm{H} 1$ promoter was PCR amplified from the complete pDECKO plasmid and was cloned into the intermediate plasmid. The reaction was transformed in Stbl 3 competent cells. Positive clones were validated using colony PCR and sequencing.

\section{Cell culture conditions.}

HEK 293 T cells were obtained from ATCC and grown in Dulbecco's Modified Eagle Medium (DMEM) supplemented with 10\% Fetal Bovine Serum (FBS) 1X Penicillin/Streptomycin (Pen Strep, catalog \# 15140122). Merkel Cell Carcinoma cell lines, MKL-1 were obtained from ECACC (\#09111801) and cultured in Roswell Park Memorial Institute (RPMI) Media with $10 \%$ FBS and $1 \mathrm{X}$ Penicillin/Streptomycin. The cells were grown at $37^{\circ} \mathrm{C}$ with $5 \% \mathrm{CO}_{2}$.

\section{Lentiviral vector construction and transduction.}

Lentiviral vectors were constructed by co-transfecting HEK 293 T cells with plasmid of interest, the Gag-Pol packaging plasmid psPAX2 (Addgene \#12259), and pVSVG (Addgene \#8454) expressing plasmid in a ratio of 5:3.75:1.25. Lipofectamine ${ }^{\circledR}$ LTX (Invitrogen \#15338100) was used in 3:1 ratio with the total amount of DNA to be transfected. DNA, Lipofectamine LTX (Invitrogen \#15338500) and OptiMEM mix were incubated at RT for 20 minutes and added to cells in antibiotics free media. Viral media was collected $48 \mathrm{~h}$ and $72 \mathrm{~h}$ post transfection. The viral media was filtered through $0.45 \mu \mathrm{m}$ filter followed by concentration through Amicon column and addition of $1 / 3^{\text {rd }}$ volume of LentiX Concentrator (Takara \#631231). Viral pellet was obtained by spin down at $1500 \mathrm{~g}$ at $4^{\circ} \mathrm{C}$, resuspended in RPMI media and added to the MKL-1 cells to be transduced. Screening of gRNAs was done by transducing pLentiCRISPRv2 plasmid containing the gRNAs in MKL-1 cells followed by puromycin $(1 \mu \mathrm{g} / \mathrm{mL})$ selection. pLentiCRISPRv2 (Addgene \#52961) without gRNA was used for 
mock transduction. For constructing MKL-1 pDECKO mCherry $\mathrm{C} 3+\mathrm{C} 13$ cell line, $\mathrm{MKL}-1$ cells were transduced with pDECKO mCherry C3+C13 plasmid. pDECKO mCherry empty vector (EV; without gRNAs) was used as an empty vector for mock transduction. MKL-1 Cas9 cell line was developed using pCWCas9 Blast (Addgene \#83481) with Blasticidin (10ug/mL) selection for 10 days post transduction. Cas9 was expressed by $1 \mathrm{ug} / \mathrm{ml}$ of Doxycycline induction.

\section{Western blot analysis.}

Cells were lysed in EBC buffer (50 mM Tris pH-8, $150 \mathrm{mM} \mathrm{NaCl}, 0.5 \mathrm{mM}$ EDTA, $\beta$ mercaptoethanol-1:10,000, 0.5\% NP-40, 1 mM PMSF supplemented with protease inhibitor cocktail (Roche, Cat \#118575). Protein lysate was quantitated using Pierce BCA protein assay kit. The lysate was denatured in 1x Laemmli buffer by boiling at $99^{\circ} \mathrm{C}$ for 5 minutes. The proteins were separated using SDS-PAGE and transferred to a polyvinylidene fluoride (PVDF) membrane (\#88518, Biorad) The PVDF membrane was blocked with 5\% Blotto (SC-2325, Santacruz) in $1 \mathrm{X}$ TBS-T. The blot was developed with primary antibodies overnight at $4^{0} \mathrm{C}$ a $\mathrm{s}$ follows: antiMCPyV Tumor antigen monoclonal antibody (1:5000;Ab3 and Ab5 were used (put citation)), anti-Cas9 monoclonal antibody (1:1000; Cell signaling \#14697), and anti-Vinculin monoclonal antibody (1:10000; Sigma \#V9131). The PVDF membrane was incubated in peroxidase-labeled secondary antibodies. The ECL ${ }^{\mathrm{TM}} \mathrm{Prime}^{\mathrm{T}}$ Western Blotting System (\#RPN2232, GE Healthcare) was used for the detection of chemiluminescence according to the manufacturer's protocol. The chemiluminescence was detected using ImageQuant ${ }^{\mathrm{TM}}$ Las 4000 (GE Healthcare).

\section{RESULTS}

To target the integrated MCPyV Tumour antigens present in MCPyV positive MCC cells, we designed 13 sgRNAs. These gRNAs are complementary to regions between 247 to 1254 nucleotides of MKL-1 genomic sequence that encompass both LT and sT (Table 1 and Supplementary Fig 1). 5 gRNAs (C1 - C5) target Exon 1 (common region between ST and LT), 4 gRNAs (C6 - C9) target only sT and the rest of the 4 gRNAs (C10 - C13) target only LT. These sgRNAs were cloned into pLentiCRISPRv2 which also expressed SpCas9 protein under the control of a U6 promoter. To investigate the cleavage activity of these 13 sgRNAs, we transduced MKL-1 cells with these 13 constructs. Immunoblotting showed significant reduction in the expression of both ST and LT by 2 gRNAs - C3 and C13 (Supplementary Figure 1). We thereby proceeded with these two gRNAs for further experimentation (Figure 1).

To further validate the cleavage efficiency of gRNAs C3 and C13, we performed in vitro cleavage assays (Figure 2). We cloned the NCCR and T antigen derived from MKL-1 into a pLentiCMV backbone vector and used the target region PCR amplicon as the DNA substrate for our assays. The two gRNAs efficiently cleaved the target substrate as shown by the cut bands of sizes 562bp, 367bp and 195bp for C3 and 512bp and 400bp for C13, respectively (Figure 2). As shown, we used two different SpCas9 enzymes as technical replicates to test our gRNAs. To address the time taken for this cleavage, we further performed a time course assay. Both the gRNAs 
were able to cleave the target within 10 minutes (Figure 3). Therefore, C3 and C13 can effectively target and cleave MCPyV T antigen genomic sequence.

Next, using in vitro cleavage assays, we validated the combinatorial target cleavage by gRNAs C3 and C13 (Figure 4). The effective cleavage activity is shown by the 3 cleaved bands at $1131 \mathrm{bp}, 940 \mathrm{bp}$ and $374 \mathrm{bp}$ as indicated by the arrows (Figure 4).

To investigate the effects of these CRISPR/Cas9 constructs in combination, we cloned C3 and C13 into pDECKO mCherry vector. This vector expresses 2 gRNAs together under $\mathrm{U} 6$ and $\mathrm{H} 1$ promoters respectively (Figure 5A).

To test the efficiency of this construct we co-transfected HEK293T cells with Merkel cell polyomavirus (MCC LT162) early region expressing plasmid, Cas9- expressing plasmid and the pDECKO mCherry empty vector (EV) or pDECKO mCherry C3 + C13. Western blot data revealed a loss of LT expression upon transfection of pDECKO C3 + C13 relative to pDECKO mCherry empty vector (Figure 5B).

Further, we targeted MCPyV T antigen locus in MKL-1 cells using C3 and C13 gRNAs using lentiviral-vector delivery. We transduced MKL-1 cells and harvested cells post-selection. We first developed stable pDECKO mCherry EV and pDECKO mCherry $\mathrm{C} 3+\mathrm{C} 13$ expressing cell lines. For this we infected MKL-1 cells with pDECKO lentiviral vectors followed by Puro selection. Additionally, we expressed Cas9 using a Tet-inducible system in these MKL-1 stable cell lines using Blasticidin selection. Infection followed by selection of MKL-1 cells with Cas9 and 2 gRNAs showed a significant reduction in MCPyV T antigen expression (Figure 5C).

\section{DISCUSSION}

Changes in DNA causes cancer, and in virally caused cancers such as MCC this change refers to the integration of a viral genome. Scientists have been searching for easy ways to correct DNA changes linked to cancer and other diseases for ages, when CRISPR came along in 2013.

The gene-editing tool CRISPR is a game changer offering a precise, customizable, fast and an easy-to-use pair of molecular scissors to cut human DNA within cells.

For MCPyV positive Merkel cell carcinoma, RNAi has already been used to interrogate the effect of blocking MCPyV $T$ antigen gene expression in cancer cells and testing phenotype ${ }^{9}$. Knocking down of $\mathrm{T}$ antigens via shRNA kill MCC cancer cell lines. The oncogenic dependency of Merkel tumor cells on $T$ antigen expression renders them essential for their survival, thus making them excellent targets for potential therapy.

Our strategy used CRISPR to target the MCPyV T antigen genomic region and cause two double stranded DNA cuts. The excision of an important DNA sequence of the viral genome region, flanking between the two target sites, by DSBs at two sites caused a disruption in gene expression. This strategy is more robust as compared to targeting via single gRNA as the excision of DNA sequence leads to knock out of target gene. Further, we validated the gRNAs in HEK293 cells stably expressing MCPyV T antigens and also in MCPyV positive MKL-1 cells and found a significant reduction in MCPyV T antigen levels. 
CRISPR using Cas9 has several advantages to RNAi; besides being more precise, it targets the genomic DNA as opposed to mRNA and causes a permanent gene disruption and thus a stronger effect.

The field of CRISPR is growing rapidly since its discovery and its application in human gene editing was established. Since we began the study, Cas13a and Cas13d have been used successfully and efficiently to target protein-coding mRNA transcripts to knock-down gene expression in human cells ${ }^{20-22}$. This Type $\mathrm{VI}$ CRISPR Cas system holds great promise for the future of gene expression loss mediated therapy ${ }^{23}$.

Our study provides a proof of principle for the use of CRISPR technology to edit out MCPyV T antigen from the Merkel tumor genome. Removal of MCPyV T antigens by using the SpCas9/sgRNA combinations system has significant clinical potential in the treatment of MCPyV positive MCC. However slipping CRISPR components into lab-grown cells in one thing, the delivery of the Cas enzymes and gRNA into a cancer patient's body remains the challenge.

In the future, this tool can be delivered in vivo using Adeno associated virus (AAV) vectors ${ }^{24}$, which are smaller and safer than lentivirus or retrovirus vectors and can infect with high titers. As AAV vectors have also been employed previously for gene therapy they could be potential vectors for in vivo delivery of Cas 9 and gRNAs ${ }^{24}$.

Recently Chen et al., 2019 developed a promising alternative CRISPR delivery system using nanocapsules ${ }^{25}$. Their $25 \mathrm{~nm}$ diameter polymer capsule can carry Cas9 and a gRNA. Using specific peptides and other ingredients, this capsule can target predetermined cell types. It is highly stable in the bloodstream and releases its contents once inside the cell ${ }^{25}$.

Researchers are continuing to explore different ways to fine-tune this delivery of CRISPR components to specific organs and cell-types of the human body ${ }^{24,26,27}$. We hope that in the near future, such a smooth delivery will enable the two gRNAs described, to be used for targeting MCPyV for MCC therapy successfully prolonging patient lives. 


\section{FIGURES}
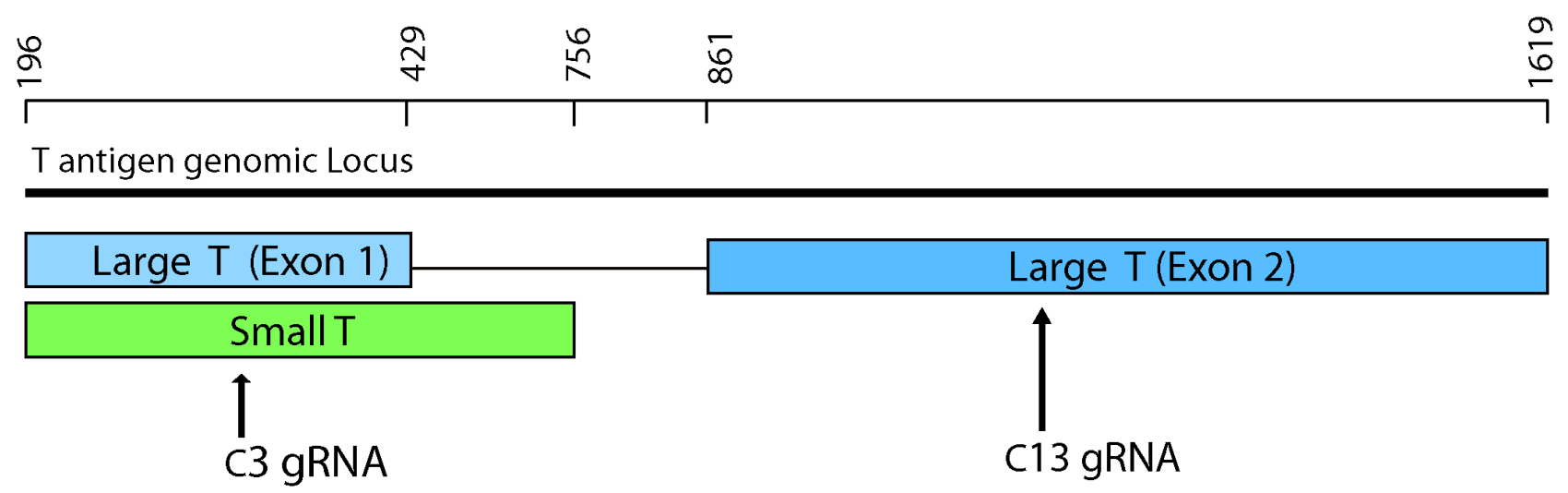

Figure 1. gRNA annotation on MCPyV T Antigens. gRNA C3 targets the genomic region encoding both the exon 1 of Large T antigen and small T antigen and gRNA C13 targets the genomic region targeting exon 2 of large $T$ antigen. A cut at both sites causes an excision of the $T$ antigen genomic region shown. 
A.

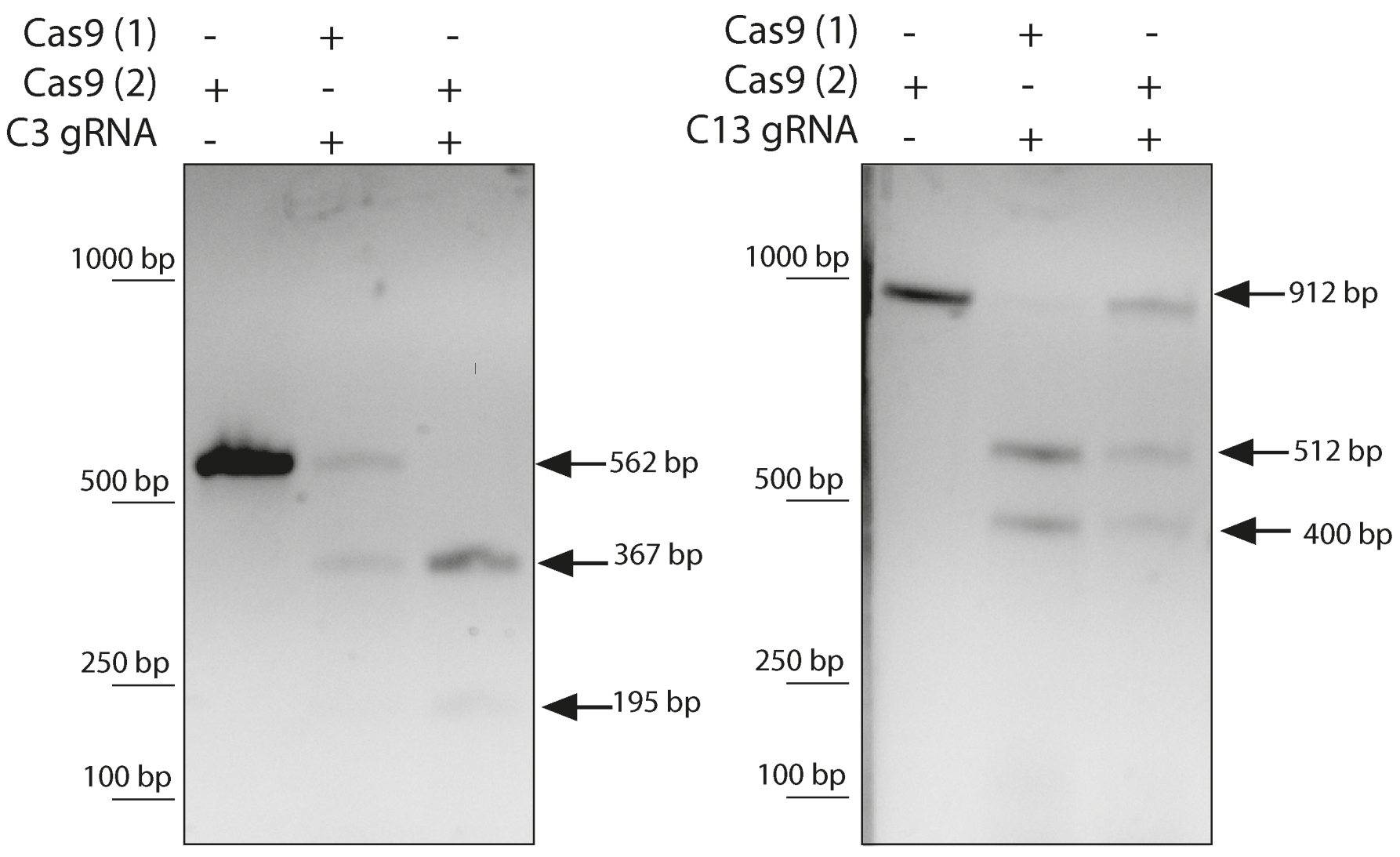

Figure 2. In vitro Cleavage Assay for gRNAs C3 and C13. The MCPyV C3 and C13 gRNA target region template was amplified from RAZ2 plasmid via PCR. In vitro cleavage reaction performed on this template shows SpCas9 mediated MCV target cleavage in the presence of gRNAs (A.) C3 and (B.) C13 as indicated by arrows. 
bioRxiv preprint doi: https://doi.org/10.1101/2021.02.14.431142; this version posted February 15, 2021. The copyright holder for this preprint (which was not certified by peer review) is the author/funder, who has granted bioRxiv a license to display the preprint in perpetuity. It is made available under aCC-BY-NC 4.0 International license.

A.

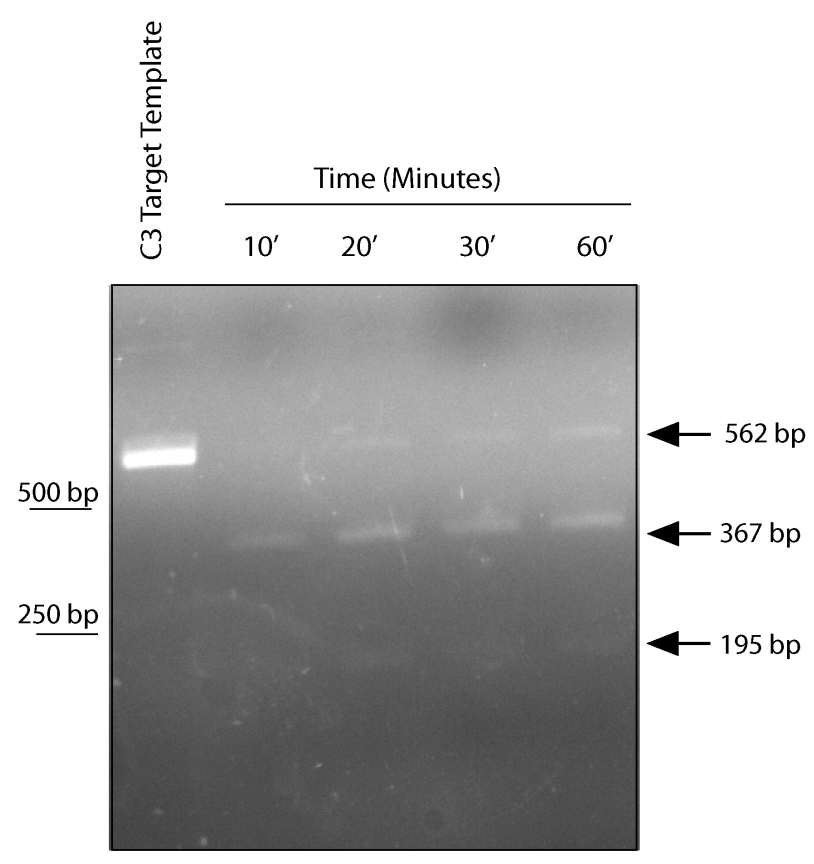

B.

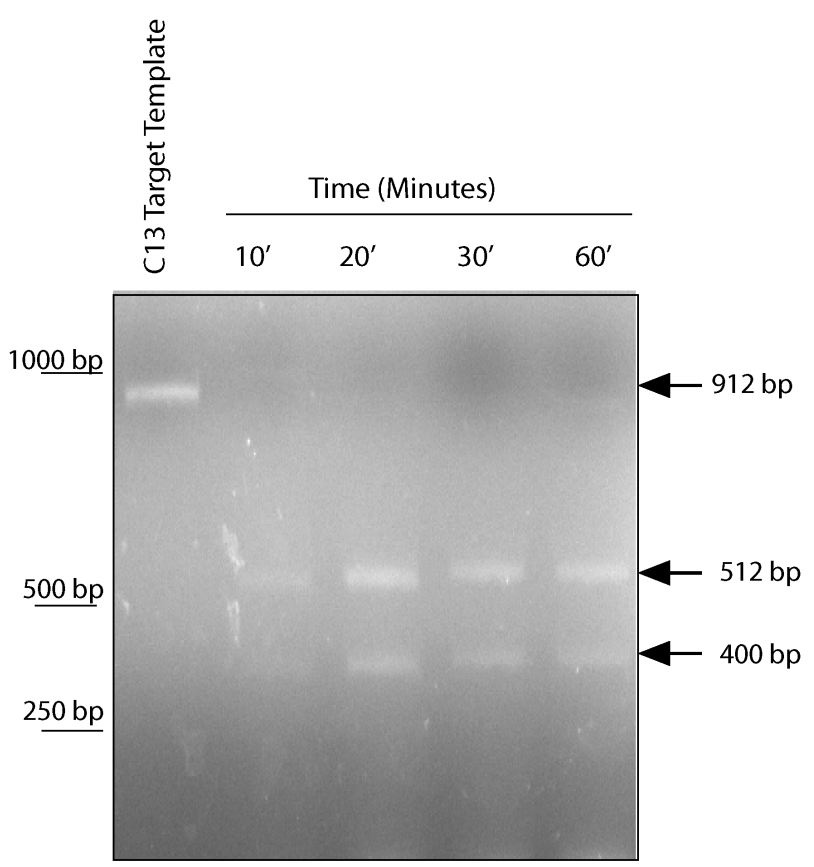

Figure 3. Time course In vitro cleavage assays for gRNAs C3 and C13. SpCas9 can cleave the MCV target template within 10 minutes. 


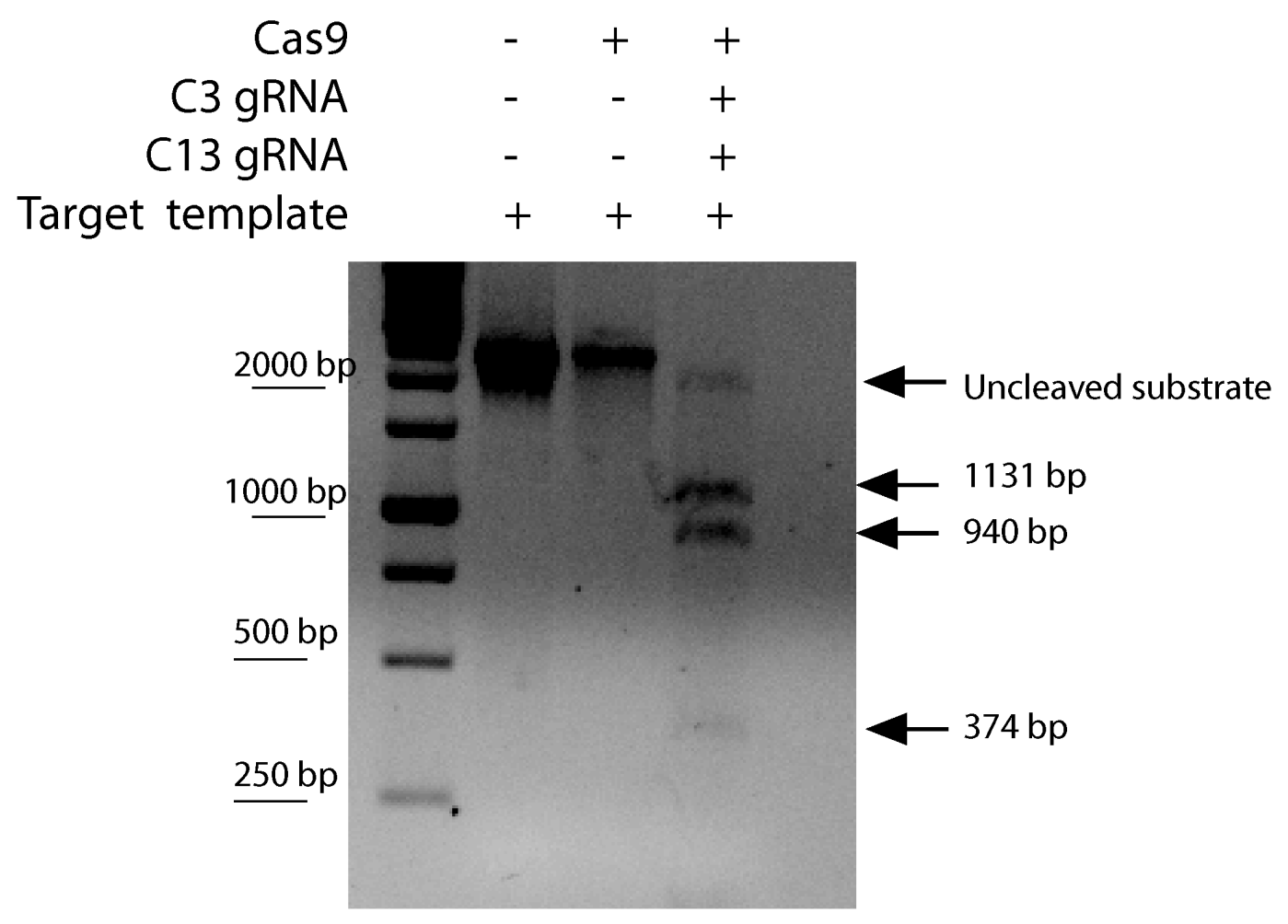

Figure 4. In vitro Cleavage Assay for gRNAs C3 and C13 together. A 2445 bp long MCV target was amplified from RAZ2 plasmid. Cas9 in the presence of C3 and C13 gRNAs led to a successful combinatorial target cleavage. 
A.

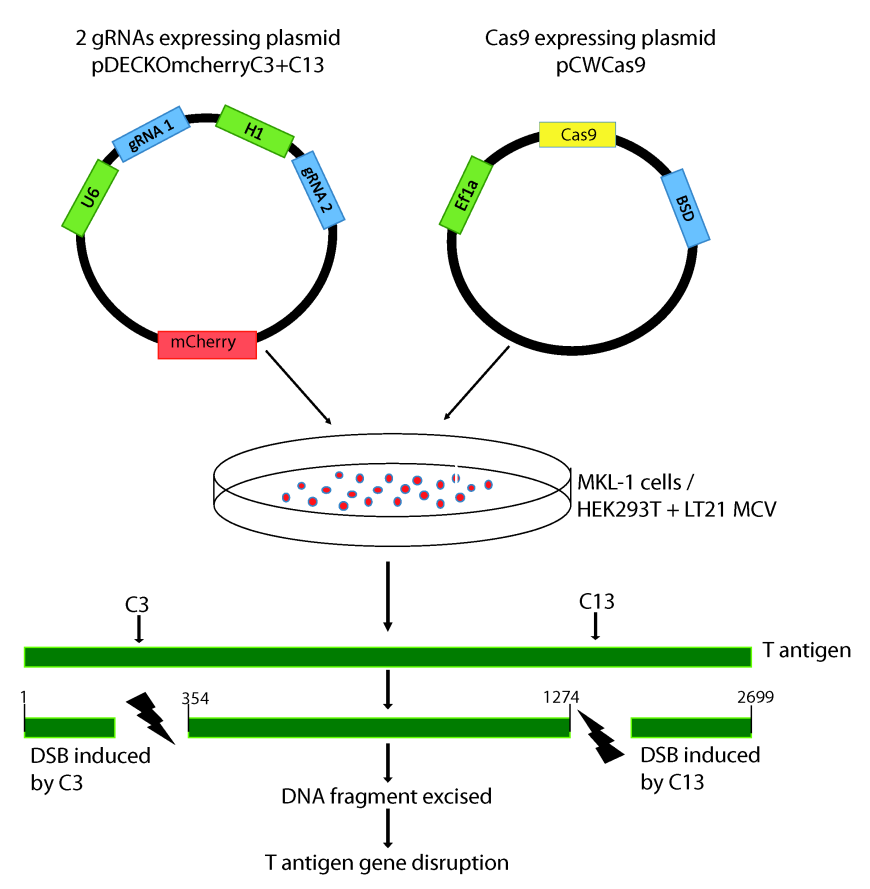

B.

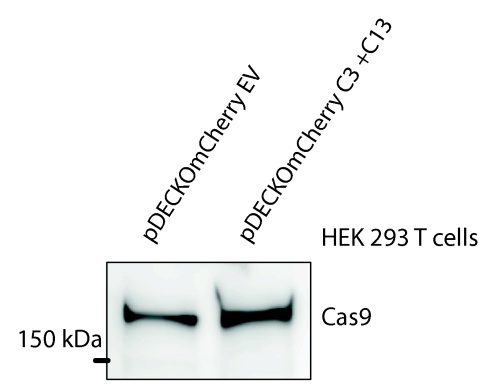

c.
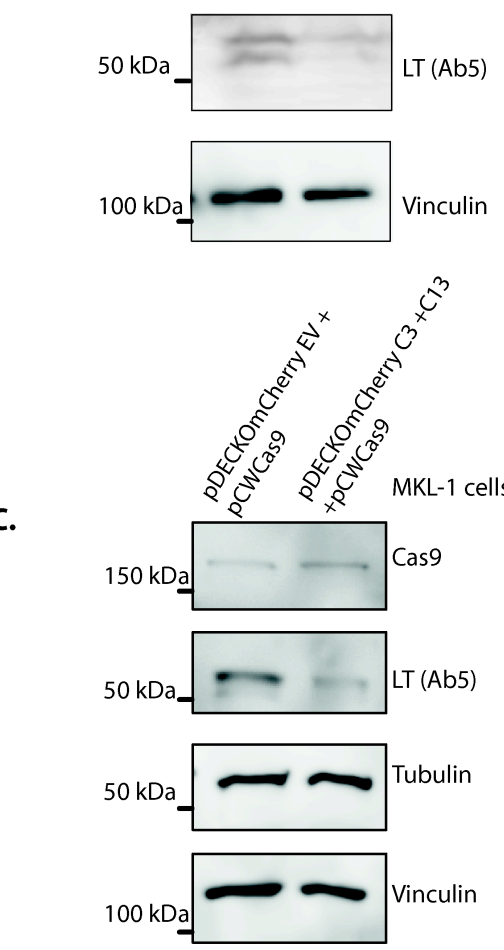

Figure 5. CRISPR Cas9 Targeting of viral protein in MCPyV T antigen expressing cells.

(A.) Schematic showing strategy for gRNA and Cas9 expression. (B.) Immunoblot showing T antigen targeting in HEK293T cells expressing both MCPyV T antigen and Cas9. (C.) Immunoblots showing T antigen loss in MKL1 cells. 


\section{FUNDING}

This work was supported by the Wellcome Trust/DBT India Alliance ( Early Career Award IA/E/14/1/501773 to $\mathrm{RA})$

\section{ACKNOWLEDGEMENTS}

We are grateful to Dr. Jingwei Cheng and Dr. James Decpario for valuable discussions and guidance. We are also very grateful to Dr. Debojyoti Chakraborty and Mohammed Azhar from IGIB, Delhi for guidance and advice on CRISPR related experiments. Special thank you to Lamiya Dohadwala and Bhavana Nayer for technical assistance and to Prof. Sanjeev Galande for scientific comments. RA would also like to thank Zaina, Ziyah, and Karan for their love, strength, and support.

\section{REFERENCES}

1. Becker, J.C., et al. Merkel cell carcinoma. Nat Rev Dis Primers 3, 17077 (2017).

2. Engels, E.A., Frisch, M., Goedert, J.J., Biggar, R.J. \& Miller, R.W. Merkel cell carcinoma and HIV infection. Lancet 359, 497-498 (2002).

3. Harms, P.W., et al. The biology and treatment of Merkel cell carcinoma: current understanding and research priorities. Nat Rev Clin Oncol 15, 763-776 (2018).

4. Feng, H., et al. Cellular and viral factors regulating Merkel cell polyomavirus replication. PLoS One 6, e22468 (2011).

5. Goh, G., et al. Mutational landscape of MCPyV-positive and MCPyV-negative Merkel cell carcinomas with implications for immunotherapy. Oncotarget 7, 3403-3415 (2016).

6. Harms, P.W., et al. The Distinctive Mutational Spectra of Polyomavirus-Negative Merkel Cell Carcinoma. Cancer Res 75, 3720-3727 (2015).

7. Starrett, G.J., et al. Merkel Cell Polyomavirus Exhibits Dominant Control of the Tumor Genome and Transcriptome in Virus-Associated Merkel Cell Carcinoma. mBio 8(2017).

8. Shuda, M., et al. T antigen mutations are a human tumor-specific signature for Merkel cell polyomavirus. Proc Natl Acad Sci U S A 105, 16272-16277 (2008).

9. Houben, R., et al. Merkel cell polyomavirus-infected Merkel cell carcinoma cells require expression of viral T antigens. J Virol 84, 7064-7072 (2010).

10. Houben, R., et al. An intact retinoblastoma protein-binding site in Merkel cell polyomavirus large $T$ antigen is required for promoting growth of Merkel cell carcinoma cells. Int J Cancer 130, 847-856 (2012).

11. Shuda, M., Chang, Y. \& Moore, P.S. Merkel cell polyomavirus-positive Merkel cell carcinoma requires viral small T-antigen for cell proliferation. J Invest Dermatol 134, 1479-1481 (2014).

12. Barrangou, R., et al. CRISPR provides acquired resistance against viruses in prokaryotes. Science 315, 1709-1712 (2007).

13. Swarts, D.C., van der Oost, J. \& Jinek, M. Structural Basis for Guide RNA Processing and Seed-Dependent DNA Targeting by CRISPR-Cas12a. Mol Cell 66, 221-233 e224 (2017).

14. Hsu, P.D., Lander, E.S. \& Zhang, F. Development and applications of CRISPR-Cas9 for genome engineering. Cell 157, 1262-1278 (2014).

15. Gupta, D., et al. CRISPR-Cas9 system: A new-fangled dawn in gene editing. Life Sci 232, 116636 (2019).

16. Cong, L., et al. Multiplex genome engineering using CRISPR/Cas systems. Science 339, 819-823 (2013). 
17. Ophinni, Y., Inoue, M., Kotaki, T. \& Kameoka, M. CRISPR/Cas9 system targeting regulatory genes of HIV-1 inhibits viral replication in infected T-cell cultures. Sci Rep 8, 7784 (2018).

18. Kennedy, E.M., et al. Inactivation of the human papillomavirus E6 or E7 gene in cervical carcinoma cells by using a bacterial CRISPR/Cas RNA-guided endonuclease. J Virol 88, 11965-11972 (2014).

19. Hu, Z., et al. Disruption of HPV16-E7 by CRISPR/Cas system induces apoptosis and growth inhibition in HPV16 positive human cervical cancer cells. Biomed Res Int 2014, 612823 (2014).

20. Zhang, C., et al. Structural Basis for the RNA-Guided Ribonuclease Activity of CRISPR-Cas13d. Cell 175, 212-223 e217 (2018).

21. Konermann, S., et al. Transcriptome Engineering with RNA-Targeting Type VI-D CRISPR Effectors. Cell 173, 665676 e614 (2018).

22. Abudayyeh, O.O., et al. C2c2 is a single-component programmable RNA-guided RNA-targeting CRISPR effector. Science 353, aaf5573 (2016).

23. Tang, L. Guiding Cas13 for RNA knockdown. Nat Methods 17, 461 (2020).

24. Wang, D., Zhang, F. \& Gao, G. CRISPR-Based Therapeutic Genome Editing: Strategies and In Vivo Delivery by AAV Vectors. Cell 181, 136-150 (2020).

25. Chen, G., et al. A biodegradable nanocapsule delivers a Cas9 ribonucleoprotein complex for in vivo genome editing. Nat Nanotechnol 14, 974-980 (2019).

26. Liu, C., Zhang, L., Liu, H. \& Cheng, K. Delivery strategies of the CRISPR-Cas9 gene-editing system for therapeutic applications. J Control Release 266, 17-26 (2017).

27. Oude Blenke, E., Evers, M.J., Mastrobattista, E. \& van der Oost, J. CRISPR-Cas9 gene editing: Delivery aspects and therapeutic potential. J Control Release 244, 139-148 (2016). 
Table 1. gRNAs targeting MCV T antigen genomic region

\begin{tabular}{|c|c|c|c|c|c|c|c|}
\hline gRNA & Sequence & Location & Strand & MIT-CRISPR Score & $\begin{array}{l}\text { Broad-Software } \\
\text { Score }\end{array}$ & $\begin{array}{l}\text { CRISPOR Specificity } \\
\text { score }\end{array}$ & $\begin{array}{c}\text { CRISPOR } \\
\text { Out of } \\
\text { frame score }\end{array}$ \\
\hline \multicolumn{8}{|c|}{ Target both large and small T } \\
\hline $\mathrm{C} 1$ & GAGATTGCTCCTAATTGTTA & Exon $1(+247$ to +266$)$ & sense & 70 & 0.3268 & 73 & 57 \\
\hline $\mathrm{C} 2$ & GGGATGTTGCCATAACAATT & Exon $1(+259$ to +278$)$ & antisense & 74 & 0.3962 & 81 & 62 \\
\hline $\mathrm{C} 3$ & AAAGCATCACCCTGATAAAG & Exon $1(+312$ to +331$)$ & sense & 69 & 0.6128 & 71 & 65 \\
\hline $\mathrm{C} 4$ & CAGGATTTCCCCCTTTATCA & Exon $1(+324$ to +343$)$ & antisense & 65 & 0.5483 & 49 & 67 \\
\hline $\mathrm{C} 5$ & TGATGGAATTGAACACCCTT & Exon $1(+350$ to +369$)$ & sense & 76 & 0.4187 & 79 & 63 \\
\hline \multicolumn{8}{|c|}{\begin{tabular}{|c|} 
Target only small T \\
\end{tabular}} \\
\hline $\mathrm{C} 6$ & GCTAGATTTTGCAGAGGTCC & Exon $1(+496$ to +515$)$ & sense & 71 & 0.3757 & 77 & 65 \\
\hline $\mathrm{C7}$ & AGTTGCTTAAGCATGCACCC & Exon $1(+517$ to +536$)$ & antisense & 83 & 0.7104 & 86 & 66 \\
\hline $\mathrm{C} 8$ & CAAAAAAACTGTCTGACGTG & Exon $1(+607$ to +626$)$ & sense & 73 & 0.6609 & 84 & 61 \\
\hline C9 & GTTTGGATTTCCTCCTACTT & Exon $1(+663$ to +682$)$ & sense & 66 & 0.3988 & 72 & 69 \\
\hline \multicolumn{8}{|c|}{$\begin{array}{ll}\text { Target only Large T } \\
\end{array}$} \\
\hline C10 & TTGACGAGGCCCCTATATAT & Exon $2(+862$ to +884$)$ & sense & 92 & 0.5301 & 95 & 73 \\
\hline C11 & AATATGGGCCCAATCCACAC & Exon $2(+943$ to +965$)$ & sense & 82 & 0.6633 & 82 & 70 \\
\hline $\mathrm{C} 12$ & TATATCGGGTCCTCTGGACT & Exon $2(+1093$ to +1115$)$ & antisense & 84 & 0.5193 & 91 & 66 \\
\hline $\mathrm{C} 13$ & TTCCAGAACGGATGGCACCT & Exon $2(+1232$ to +1254 & sense & 76 & 0.5995 & 80 & 69 \\
\hline
\end{tabular}


Supplementary Table 1. Primers Used

\begin{tabular}{|c|c|c|c|}
\hline S. No & & Description & Sequence $\left(5^{\prime}-3^{\prime}\right)$ \\
\hline \multicolumn{4}{|c|}{ Oligonucleotides for C3 and C13 in vitro transcription } \\
\hline & 1 & C3 IVT OLIGO F & TAATACGACTCACTATAGGGAAAGCATCACCCTGATAAAGGTTTTAGAGCTAGAA \\
\hline & 2 & C13 IVT OLIGO F & TAATACGACTCACTATAGGGTTCCAGAACGGATGGCACCTGTTTTAGAGCTAGAA \\
\hline & 3 & gRNA scaffold IVT oligo $\mathrm{R}$ & AAAAGCACCGACTCGGTGCCACTTTTTTCAAGTTGATAACGGACTAGCCTTATTTTAACTTGCTATTTCTAGCTCTAAAAC \\
\hline \multicolumn{4}{|c|}{ Primers for PCR amplifying Cas9 MCV IVC template } \\
\hline & 4 & IVC RAZ1 target template F & TTGGCTGCCTAGGTGACTTT \\
\hline & 5 & IVC RAZ1 target template R & GTGGATGTGGAATGTGTGCG \\
\hline \multicolumn{4}{|c|}{ Oligonucleotides for Gibson cloning of C3 and C13 in pDECKO mCherry } \\
\hline & 6 & C3/C13.1R_Gib & TCAGGGTGATGCTTTCCGGTTTCGTCCTTTCCACAAGAT \\
\hline & 7 & C3/C13.2F_Gib & ACCGGAAAGCATCACCCTGATAAAGGTTTTAGAGCTAGAAGAGAC \\
\hline & 8 & C3/C13.3R_Gib & GAGACGGGATCCTAGGAATTCCGTCTCTTCTAGCTCTAAAAC \\
\hline & 9 & C3/C13.4F_Gib & TTCCTAGGATCCCGTCTCTCTGTATGAGACCACTCTTTCCC \\
\hline & 10 & C3/C13.5R_Gib & AACAGGTGCCATCCGTTCTGGAAGGGAAAGAGTGGTCTCAT \\
\hline & 11 & C3/C13.6F_Gib & GAACGGATGGCACCTGTTTTAGAGCTAGAAATAGCAAGTT \\
\hline & 12 & Scaffold F & GTTTTAGAGCTAGAAATAGCAAG \\
\hline & 13 & $\mathrm{H} 1 \_\mathrm{R}$ & GTGGTCTCATACAGAACTTATAAG \\
\hline \multicolumn{4}{|c|}{ Primers for Cas9 target region PCR from MKL-1 genomic DNA } \\
\hline & 14 & C3 target amplicon F & TTGGCTGCCTAGGTGACTTT \\
\hline & 15 & C3 target amplicon $\mathrm{R}$ & CCAGGACCTCTGCAAAATCT \\
\hline & 16 & C13 target amplicon F & TGCTTACTGCATCTGCACCT \\
\hline & 17 & C13 target amplicon $\mathrm{R}$ & AAATGGCAAAACAACTTACTGTT \\
\hline
\end{tabular}




\section{Supplementary Figure 1}

A.

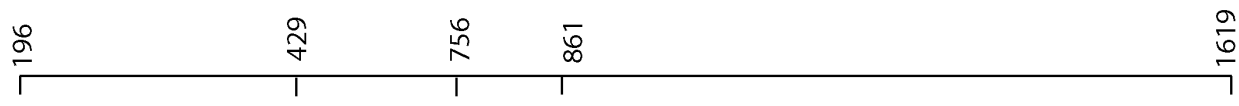

B.

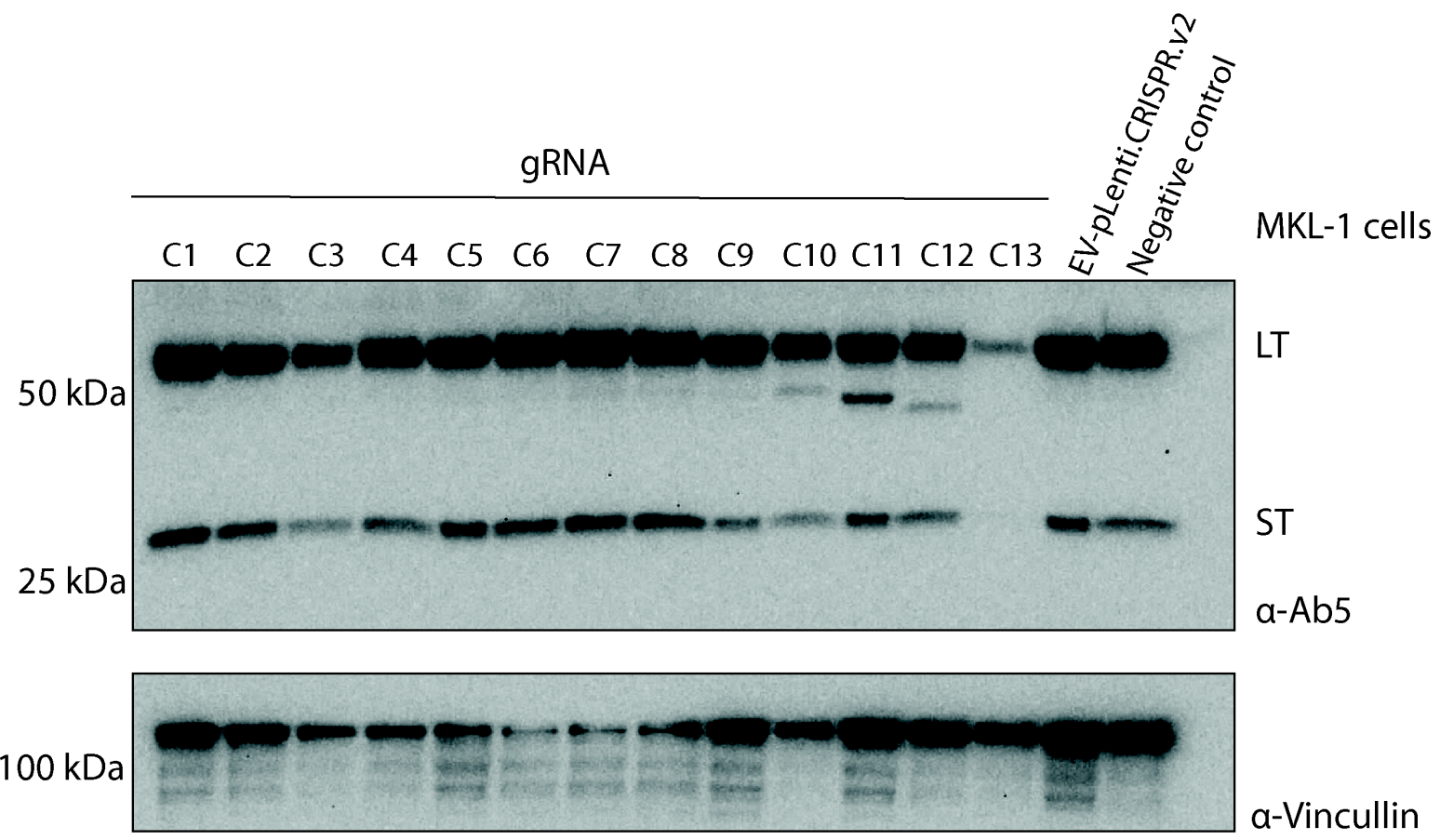

Figure S1. gRNAs target MCV T antigen. (A.) 13 gRNAs were designed to target MCPyVT antigen. (B.)To investigate the cleavage activity of these 13 sgRNAs, MKL-1 cells was transduced with 13 gRNA plasmid constructs. Immunoblotting above shows significant reduction in the expression of both ST and LT by 2 gRNAs - C3 and C13. Ab5 was used to measure MCPyVT antigen expression and Vincullin was used as a housekeeping control. The empty vector pLenti.CRISPR.v2 and only MKL-1 cell lysate was used as negative controls. 